\title{
Using Canonical Correlations in Testing for Common Nonlinear Components
}

\author{
Alenka Kavkler ${ }^{1}$ and Bernhard Böhm ${ }^{2}$
}

\begin{abstract}
The paper discusses the use of canonical correlations for modelling multiple equation systems with common nonlinear components of a smooth transition type. The coefficients in a smooth transition regression model are assumed to be nonlinear continuous functions of a properly chosen transition variable. The obtained model thus captures structural changes in the relationship between the observed economic variables. With the help of the canonical correlations technique a simple test for testing for common nonlinear components helps us interpret the relationships between different economic variables and also simplifies the specification and estimation of the equation system, since in this case a reduction in the dimension of nonlinear components is possible. It was shown by Anderson and Vahid (1998) that the test for common nonlinearities is related to the test statistic for the overidentifying restrictions in the generalized method of moments framework and that both tests have the same asymptotic distribution.

As an application of the described modelling approach, a three-variable linear vector autoregressive (VAR) model of the consumer price index for Slovenia and Austria and the nominal exchange rate between the currencies of both countries is investigated. A single common nonlinear component is detected in all 3 equations and the estimated multivariate logistic smooth transition autoregressive model is discussed.
\end{abstract}

\section{Introduction}

This paper is devoted to an investigation of small nonlinear dynamic systems. An important problem in the identification process of economic systems is usually related to the question whether the model can be kept linear or whether nonlinear features are so dominant that they must be considered in the specification. From recent studies of univariate models one has learned that there is much to be gained by exploring such nonlinear features. Representation of asymmetric reactions, structural changes and other phenomena of economic developments can be fruitfully investigated by nonlinear modelling techniques. As many issues in economics require the specification of several relationships, techniques

\footnotetext{
${ }^{1}$ Department of Quantitative Economic Analysis, Faculty of Economics and Business, Maribor, Slovenia; alenka.kavkler@uni-mb.si

${ }^{2}$ Institute of Mathematical Methods in Economics, Vienna University of Technology, Vienna, Austria; bernhard.boehm@tuwien.ac.at
} 
to handle nonlinear features in systems are required. During the recent years such methods have appeared in the literature. We shall discuss the use of canonical correlations for modelling multiple equation systems with common nonlinear components of a smooth transition type. The coefficients in a smooth transition regression model are assumed to be nonlinear continuous functions of a properly chosen transition variable. The obtained model thus captures structural changes in the relationship between the observed economic variables. The current investigation attempts to apply these techniques to a small model of the real exchange rate, decomposed into its three components, domestic prices, foreign prices and the nominal exchange rate. The procedure to explore the appropriate specification starts with testing for common nonlinearities. Section two shortly describes the approach going back to Anderson and Vahid (1998). Given the results of this test, we continue to attempt estimation of the vector autoregressive system considering the implied restrictions not only from nonlinearity testing but also from possible cointegration. In section three we shall apply this model to the data of the Slovenian Tolar versus the Austrian Schilling, respectively Euro. Finally, we shortly reflect on similar approaches for other countries.

\section{Test for common nonlinearities}

Anderson and Vahid (1998) describe a generalized method of moments test for common nonlinear components in multiple time series. The number of nonlinear functions that need to be estimated can be reduced if the system contains common nonlinear components. The basic idea behind their work is to detect all linear combinations of the (possibly nonlinear) variables that do not exhibit nonlinear properties. The number of such linear combinations determines the number of common nonlinear components. The usual statistic and econometric tests are adapted to meet the needs of multivariate systems. The canonical correlation procedure is used to obtain the estimates of the linear combinations without the nonlinear properties.

\subsection{Definition of common nonlinearity}

Suppose that the conditional mean of the $i$-th component of an $n$-dimensional vector $y_{t}$ given a $k$-dimensional vector $x_{t}$ can be written as

$$
E\left(y_{i t} \mid x_{t}\right)=\beta_{i}^{\prime} x_{t}+\psi_{i}\left(x_{t}, \theta_{i}\right), \quad i=1, \ldots, n,
$$

where the function $\psi_{i}$ is nonlinear in $x_{t}$ and possibly also in the parameter $\theta_{i}$. If one can find $s<n$ linearly independent linear combinations of the components of the vector $y_{t}$ with a linear conditional mean, then there exists an $n \times s$ matrix $A$ with a full column rank for which the equation

$$
A^{\prime} \psi\left(x_{t}, \theta\right)=0
$$

holds. $\psi$ and $\theta$ stand for the vectors $\left(\psi_{1}, \psi_{2}, \ldots, \psi_{n}\right)^{\prime}$ and $\left(\theta_{1}, \theta_{2}, \ldots, \theta_{n}\right)^{\prime}$, respectively. Obviously, $A$ is not unique. As the matrix $A \cdot H$, where $H$ is any $s \times s$ nonsingular matrix, also satisfies the previous equation, the matrix $A$ can be normalized without loss 
of generality. A particularly useful normalization contains an $s \times s$ identity matrix as the first block. The matrix $\psi$ is partitioned analogously:

$$
A=\left[\begin{array}{c}
I_{s} \\
A^{* *}
\end{array}\right], \quad \psi\left(x_{t}, \theta\right)=\left[\begin{array}{c}
\psi^{*}\left(x_{t}, \theta\right) \\
\psi^{* *}\left(x_{t}, \theta\right)
\end{array}\right]
$$

From $A^{\prime} \psi\left(x_{t}, \theta\right)=0$ follows $\psi^{*}\left(x_{t}, \theta\right)=-A^{* *^{\prime}} \psi^{* *}\left(x_{t}, \theta\right)$ and

$$
\psi\left(x_{t}, \theta\right)=\left[\begin{array}{c}
-A^{* *^{\prime}} \\
I_{n-s}
\end{array}\right] \psi^{* *}\left(x_{t}, \theta\right)
$$

Finally, we can eliminate $s$ nonlinear components and therefore write the conditional expectation of the vector $y_{t}$ in terms of only $n-s$ nonlinear components:

$$
E\left(y_{t} \mid x_{t}\right)=B x_{t}+A^{\perp} \psi^{* *}\left(x_{t}, \theta\right)
$$

with $A^{\perp}=\left[\begin{array}{c}-A^{* *^{\prime}} \\ I_{n-s}\end{array}\right]$ and $B$ equal to the $n \times k$ matrix of stacked vectors $\beta_{i}^{\prime}, i=1, \ldots, n$.

Definition 2.1 We say that system (2.1) has $n-s$ common nonlinear components, if it is possible to rewrite (2.1) in the form (2.2) and if $s$ is the largest integer with this property.

\subsection{Smooth transition regression models}

Since we would like to study common nonlinear components of a smooth transition type, we shall start with a short description of smooth transition regression (STR) models.

\subsubsection{Univariate smooth transition regression models and the linearity test}

Many elements of economic theory mention the idea that the economy behaves differently if values of certain variables lie in one region rather than in another, or, in other words, follow different regimes. The first attempt at modelling such phenomena is represented by discrete switching models, where a finite number of different regimes is assumed. The central tool of this class of models is the so-called switching variable that can be either observable or unobservable. As smooth transition between regimes is often more convenient and realistic than just the sudden switches, several scientists proposed a generalisation of discrete switching models of the form

$$
y_{t}=x_{t}^{\prime} \varphi+\left(x_{t}^{\prime} \theta\right) \cdot G\left(\gamma, c ; s_{t}\right)+u_{t}, \quad t=1,2, \ldots, T,
$$

where

$$
x_{t}=\left(1, x_{t 1}, x_{t 2}, \ldots, x_{t k}\right)^{\prime}=\left(1, y_{t-1}, \ldots, y_{t-m}, z_{t 1}, \ldots, z_{t l}\right)^{\prime}
$$

is the vector of explanatory variables containing lags of the endogenous variable $y_{t}$ and the exogenous variables $z_{t 1}, \ldots, z_{t l}, \varphi=\left(\varphi_{0}, \varphi_{1}, \ldots, \varphi_{k}\right)^{\prime}$ and $\theta=\left(\theta_{0}, \theta_{1}, \ldots, \theta_{k}\right)^{\prime}$ are the parameter vectors and $u_{t}$ is a sequence of independent identically distributed errors. $G$ denotes a continuous transition function, usually bounded between 0 and 1 . Because of this property not only the two extreme states can be explained by the model, but also a continuum of states that lie between those two extremes. The slope parameter $\gamma$ is an 
indicator of the speed of transition between 0 and 1, whereas the threshold parameter $c$ points to where the transition takes place. The transition variable $s_{t}$ is usually one of the explanatory variables or the time trend. One of the most popular functional forms of the transition function is the logistic function

$$
G_{1}\left(\gamma, c ; s_{t}\right)=\frac{1}{1+e^{-\gamma\left(s_{t}-c\right)}}, \quad \gamma>0
$$

In this case, we refer to model (2.3) as the logistic smooth transition regression model, shortly LSTR model.

To test the null hypothesis of linearity against the alternative of an LSTR model, we first redefine the logistic transition function as $G_{1}^{*}=G_{1}-\frac{1}{2}$. The advantage of $G_{1}^{*}$ over $G_{1}$ lies in the fact that $G_{1}^{*}$ takes the value zero when $\gamma=0$, while the functional form of the STR model stays the same (only the parameter vectors $\varphi$ and $\theta$ change). The null hypothesis of linearity for model (2.3) with $G_{1}$ replaced by $G_{1}^{*}$ can be expressed as

$$
H_{0}: \gamma=0 \quad \text { against } \quad H_{1}: \gamma>0
$$

or as

$$
H_{0}^{\prime}: \theta=0 \quad \text { against } \quad H_{1}^{\prime}: \theta \neq 0 .
$$

Note that some of the parameters are not identified under the null hypothesis. In case of (2.5), this goes for the parameters $c$ and $\theta$, while in case of null hypothesis (2.6), neither $c$ nor $\gamma$ is identified. These nuisance parameters are not present in the model under $H_{0}$ and their values do not influence the value of the log-likelihood. Consequently, the standard statistical theory with the battery of likelihood ratio, Lagrange multiplier and Wald test is not applicable, since the asymptotic distributions are nonstandard. Critical values could only be determined with the help of the simulation methods. To overcome this problem, Luukkonen, Saikkonen and Teräsvirta (1998b) replaced the transition function in equation (2.3) with its third-order Taylor approximation around $\gamma=0$. After rearranging the terms one obtains

$$
y_{t}=x_{t}^{\prime} \eta_{0}+\left(\tilde{x}_{t}^{\prime} s_{t}\right) \eta_{1}+\left(\tilde{x}_{t}^{\prime} s_{t}^{2}\right) \eta_{2}+\left(\tilde{x}_{t}^{\prime} s_{t}^{3}\right) \eta_{3}+u_{t}^{*},
$$

with $\tilde{x}_{t}=\left(x_{t 1}, x_{t 2}, \ldots, x_{t k}\right)^{\prime}$. Since the transition variable is usually one of the explanatory variables, the constant was dropped from the vector $x_{t}$ to avoid duplicating the variable $s_{t}$ on the right-hand side of auxiliary regression (2.7). The procedure is explained in detail in Teräsvirta (1998). Thus, the former null hypothesis of linearity $H_{0}: \gamma=0$ implies

$$
H_{0}^{\prime \prime}: \eta_{j}=0, \forall j \in\{1,2,3\} \quad \text { and } \quad H_{1}^{\prime \prime}: \exists j \ni: \eta_{j} \neq 0,
$$

which can be tested by performing a Lagrange multiplier (LM) test. Under the null hypothesis, the statistic has an asymptotic $\chi^{2}$-distribution with $3 k$ degrees of freedom, when the so-called regularity conditions are fulfilled. Basically, the moments used in the LM test statistic have to exist (see White (2001) for details). The test has the advantage that the estimation of the nonlinear model under the alternative hypothesis is not necessary. Nevertheless, Teräsvirta (1998) recommends using the F-statistic instead. He argues that the F-statistic has better small sample properties, since its empirical size is close to the nominal size. 


\subsubsection{Multivariate STAR models}

Whereas there has been extensive research in the field of univariate nonlinear modelling, the statistical theory of multivariate nonlinear models has yet to be developed. The first attempts at extending nonlinear smooth transition regression techniques to a multivariate setting can be found in Weise (1999), van Dijk (1999) and Camacho (2004). Similarly, multivariate Markov switching models are treated in Krolzig (1997) and multivariate threshold models in Tsay (1998). Most of the work has been done with the multivariate autoregressive models.

Definition 2.2 (Anderson and Vahid) A multivariate smooth transition autoregressive model, or a STAR(p) model, is a model of the form

$$
y_{t}=A_{0}+A_{1}(L) y_{t}+F\left(z_{t}\right)\left[B_{0}+B_{1}(L) y_{t}\right]+\varepsilon_{t},
$$

where $y_{t}$ is an n-vector time series, $A_{0}$ and $B_{0}$ are $n$-vectors of constants, $A_{1}(L)$ and $B_{1}(L)$ are $p$-th order matrix polynomials in the lag operator with $A_{1}(0)=B_{1}(0)=0, \varepsilon_{t}$ is an $n \times 1$ i.i.d. $(0, \Sigma)$ sequence, $F$ is an $n \times n$ diagonal transition matrix with a typical diagonal entry $F_{i}\left(z_{i t}\right)$, and the transition variable $z_{i t}$ is one of the np lagged regressors from the vector

$$
\tilde{y}_{t}=\left(y_{t-1}^{\prime}, y_{t-2}^{\prime}, \ldots, y_{t-p}^{\prime}\right)^{\prime} .
$$

The specification that each transition function $F_{i}\left(z_{i t}\right)$ is a logistic function of the form

$$
F_{i}\left(z_{i t}\right)=\left(1+\exp \left[-\gamma_{i}\left(z_{i t}-c_{i}\right)\right]\right)^{-1},
$$

where $\gamma>0$, leads to a multivariate LSTAR model.

In the study of univariate LSTAR models by Lukkonen, Saikkonen and Teräsvirta (1998b) and Teräsvirta (1994), the models with $z_{i t}=y_{i t-d}$ are discussed. As already explained, the linearity test is developed with the help of the Taylor approximation of the transition function. The procedure is similar in the multivariate framework. The null hypothesis of linearity, i.e.

$$
H_{0}: \beta_{2 j}=\beta_{3 j}=\beta_{4 j}=0, \quad j=1, \ldots, n p,
$$

based on the auxiliary regression

$$
y_{i t}=\beta_{0}+\beta_{1}^{\prime}\left(\tilde{y}_{t}\right)+\beta_{2}^{\prime}\left(\tilde{y}_{t} \cdot z_{i t}\right)+\beta_{3}^{\prime}\left(\tilde{y}_{t} \cdot z_{i t}^{2}\right)+\beta_{4}^{\prime}\left(\tilde{y}_{t} \cdot z_{i t}^{3}\right)+v_{i t},
$$

is tested against the alternative hypothesis of a multivariate STAR model. An LM-type test can be used, as before.

\subsection{The common nonlinearities test}

Since the common nonlinearities test is deduced from the test of overidentifying restrictions in the generalized method of moments (GMM) framework, it has an asymptotic $\chi^{2}$-distribution provided that some regularity conditions described below hold. A thorough discussion of the necessary and sufficient conditions is given in Wooldridge (1994). Regularity conditions are: 
1. The variables are essentially stationary and weekly dependent.

2. The assumptions GMM.1 to GMM.10 (as defined in Wooldridge) hold.

These conditions guarantee that the GMM estimator exists, is consistent and asymptotically normally distributed.

Suppose that the null hypothesis of linearity is rejected for every component of the $n$-dimensional vector $y_{t}$. In this case, the conditional mean of $y_{i t}, i=1, \ldots, n$, is of the form (2.1). If there is a linear combination $\alpha_{1}^{\prime} y_{t}$ of the components of the vector $y_{t}$ with a linear conditional mean, then the number of nonlinear components in the system can be reduced. A reduction of this kind requires that

$$
E\left(\left(\alpha_{1}^{\prime} y_{t}^{\dagger}\right) \otimes w_{t}^{\dagger}\right)=0
$$

where $\otimes$ denotes the Kronecker product. Note that $y_{t}^{\dagger}$ was obtained from $y_{t}$ by removing the part that is linear in $x_{t} . w_{t}^{\dagger}$ stands for the $m$-dimensional vector of nonlinear regressors in the corresponding univariate linearity test. In case of a STAR model under the alternative, $w_{t}^{\dagger}$ is the vector of nonlinear regressors in auxiliary regression (2.10), namely $\left(\left(\tilde{y}_{t} \cdot z_{t}\right)^{\prime},\left(\tilde{y}_{t} \cdot z_{t}^{2}\right)^{\prime},\left(\tilde{y}_{t} \cdot z_{t}^{3}\right)^{\prime}\right)^{\prime}$. Because of the structure of equation (2.11), we can use the generalized method of moments (GMM) to estimate the vector $\alpha_{1}$. By replacing the moment condition with the corresponding sample mean $\frac{1}{T} \sum_{t=1}^{T}\left(\left(\alpha_{1}^{\prime} y_{t}^{\dagger}\right) \otimes w_{t}^{\dagger}\right)$, we can construct an objective function from the class of minimum distance estimators, see Greene (2003):

$$
\begin{aligned}
Q & =\frac{1}{T} \sum_{t=1}^{T}\left(\left(\alpha_{1}^{\prime} y_{t}^{\dagger}\right) \otimes w_{t}^{\dagger}\right)^{\prime} \times \hat{V}_{1 T}^{-1} \times \frac{1}{T} \sum_{t=1}^{T}\left(\left(\alpha_{1}^{\prime} y_{t}^{\dagger}\right) \otimes w_{t}^{\dagger}\right) \\
& =\frac{1}{T^{2}} \alpha_{1}^{\prime} Y^{\prime} W \hat{V}_{1 T}^{-1} W^{\prime} Y \alpha_{1}
\end{aligned}
$$

While $\mathrm{Y}$ and $\mathrm{W}$ denote the matrices with stacked vectors $y_{t}^{\dagger}$ and $w_{t}^{\dagger}$ as rows, $\hat{V}_{1 T}$ stands for a matrix that complies with the condition

$$
\operatorname{plim}_{T \rightarrow \infty} \hat{V}_{1 T}=\lim _{T \rightarrow \infty} E\left(T^{-1}\left(W^{\prime} Y \alpha_{1} \alpha_{1}^{\prime} Y^{\prime} W\right)\right) .
$$

The notation plim is used in econometrics to denote the probability limit. Recall that a sequence of random variables $\left\{x_{n}\right\}$ converges in probability to a random variable $x$, if $\lim _{n \rightarrow \infty} P\left(\left|x_{n}-x\right| \geq \varepsilon\right)=0$ for every $\varepsilon>0$.

The question we have to answer is how to estimate the rank and the basis of the space of vectors that satisfy equation (2.11). Let the matrix $\alpha$ consist of $s$ such vectors, $\alpha_{1}, \alpha_{2}, \ldots, \alpha_{s}$, written as columns. If the operator $\operatorname{vec}(A)$ concatenates the columns of the given matrix $A$ into one column vector, then the objective function for estimating the matrix $\alpha$ with the generalized method of moments is of the form

$$
Q=\frac{1}{T^{2}} \operatorname{vec}^{\prime}(Y \alpha)\left(I_{s} \otimes W\right) \hat{V}_{T}^{-1}\left(I_{s} \otimes W^{\prime}\right) \operatorname{vec}(Y \alpha),
$$

where

$$
\operatorname{plim}_{T \rightarrow \infty} \hat{V}_{T}=\lim _{T \rightarrow \infty} E\left[\frac{1}{T}\left(I_{s} \otimes W^{\prime}\right) \operatorname{vec}(Y \alpha) \operatorname{vec}^{\prime}(Y \alpha)\left(I_{s} \otimes W\right)\right]
$$


Under the assumption that there is no serial correlation and no heteroscedasticity,

$$
\hat{V}_{T}=\hat{\Sigma} \otimes \frac{W^{\prime} W}{T}
$$

turns out to be a suitable choice, with $\hat{\Sigma}$ equal to a consistent estimate of $E\left(\alpha^{\prime} y_{t}^{\dagger} y_{t}^{\dagger^{\prime}} \alpha\right)$. The common nonlinearities test is performed in several steps:

1. Calculate $\hat{\Sigma}$;

2. Determine the rank of $\alpha$ in a loop. Set $s$ to 0 . While overidentifying restrictions are not rejected, do the following:

a. increase $s$ by 1 ,

b. perform the GMM estimation,

c. test the overidentifying restrictions.

The number of common nonlinear components is equal to $n-s$. The identifying restrictions are carried out by setting $\hat{\Sigma}$ equal to the identity matrix, which also simplifies the procedure, as shown by the next lemma.

The common nonlinearities test is performed with the help of the canonical correlations approach. Recall that given an $n_{1}$-dimensional random vector $z_{1}$ and an $n_{2^{-}}$ dimensional random vector $z_{2}$, the first pair of canonical variables is defined as the pair of linear combinations $u_{1}=\delta_{1}^{\prime} z_{1}$ and $v_{1}=\eta_{1}^{\prime} z_{2}$ of unit variance, for which the correlation $\operatorname{Corr}\left(u_{1}, v_{1}\right)$ is maximal. Analogously, the $k$-th pair of canonical variables is the pair od linear combinations $u_{k}=\delta_{k}^{\prime} z_{1}$ and $v_{k}=\eta_{k}^{\prime} z_{2}$ with unit variance and uncorrelated with the first $k-1$ pairs of canonical variables, which maximizes the correlation $\operatorname{Corr}\left(u_{k}, v_{k}\right)$. If $n_{1} \leq n_{2}$, then there exist $n_{1}$ pairs of canonical variables. The correlation $\operatorname{Corr}\left(u_{k}, v_{k}\right)$ is the $k$-th canonical correlation and the vectors $\delta_{k}$ and $\eta_{k}$ are called the $k$-th canonical coefficient vectors of the random vectors $z_{1}$ and $z_{2}$, respectively.

Let us denote the estimated canonical correlations between the $n$-dimensional random vectors $y_{t}^{\dagger}$ and $w_{t}^{\dagger}$ by

$$
\left\{\lambda_{i}, i=1, \ldots, n\right\}
$$

where $\left\{\lambda_{i}\right\}$ are assumed to be ordered from the smallest to the largest.

\section{Lemma 2.3 (Anderson and Vahid, 1998) Under the normalization}

$(1 / T) \alpha^{\prime} Y^{\prime} Y \alpha=I_{s}$, the following holds:

1. The generalized method of moments estimators of the columns of the matrix $\alpha$ are the canonical coefficient vectors of $y_{t}^{\dagger}$ corresponding to

$$
\left\{\lambda_{i}, i=1, \ldots, s\right\}
$$

the s smallest estimated canonical correlations between $y_{t}^{\dagger}$ and $w_{t}^{\dagger}$.

2. The test statistic for the overidentifying restrictions can be computed as $J=T \sum_{i=1}^{s} \lambda_{i}^{2}$. 
The proof is a straightforward application of the Lagrange multiplier technique (see Anderson and Vahid (1998) for details). As already mentioned, the regularity conditions guarantee the asymptotic $\chi^{2}$-distribution of the test statistic $J$. The degree of freedom is equal to the number of overidentifying restrictions, in our case $(m-n) s+s^{2}$. Observe that the number of moment conditions in

$$
E\left(\left(\alpha_{j}^{\prime} y_{t}^{\dagger}\right) \otimes w_{t}^{\dagger}\right)=0, \quad j=1,2, \ldots, s,
$$

is equal to $m s$, whereas the number of parameters (i.e. components of the matrix $\alpha$ ) to be estimated is $n s-s^{2}$, since $s^{2}$ parameters are determined by normalization. Therefore, the number of overidentifying restrictions is equal to $m s-\left(n s-s^{2}\right)$.

\subsection{Testing for common STAR nonlinearities}

Suppose that in the multivariate STAR setting the univariate LSTAR test is applied to $y_{1}, y_{2}, \ldots, y_{n}$, every time with the same transition variable $z_{t}$. If the test rejects the null hypothesis of linearity for at least two variables, say $y_{i}$ and $y_{j}$, it is possible that the variables share a common nonlinearity. In this case one can find at least one $n$-dimensional vector $\alpha_{1}$ for which the condition (2.11) is fulfilled:

$$
E\left(\left(\alpha_{1}^{\prime} y_{t}^{\dagger}\right) \otimes w_{t}^{\dagger}\right)=0
$$

with $w_{t}$ equal to $\left(\left(\tilde{y}_{t} \cdot z_{t}\right)^{\prime},\left(\tilde{y}_{t} \cdot z_{t}^{2}\right)^{\prime},\left(\tilde{y}_{t} \cdot z_{t}^{3}\right)^{\prime}\right)^{\prime}$. The sign $\dagger$ indicates that the influence of the linear terms, namely the constant and the $\tilde{y}_{t}$, has been removed from the vectors $w_{t}$ and $y_{t}$ by regressing them on the constant and the components of the vector $\tilde{y}_{t}$. Taking into account the results from Lemma 2.3, the test statistic for the null hypothesis that there are at least $s$ linearly independent linear combinations of the components of the vector $y_{t}$ with a linear conditional mean is of the form

$$
J=T \sum_{i=1}^{s} \lambda_{i}^{2} .
$$

The $\lambda_{i}$ 's are the estimated canonical correlations between the random vectors $y_{t}^{\dagger}$ and $w_{t}^{\dagger}$. Obviously, if there are $s$ independent linear combinations with a linear mean in a model where all $n$ dependent variables are nonlinear in mean, then there are $n-s$ common nonlinear components. The null hypothesis is rejected when there are more than $n-s$ common nonlinear components. Provided that the described regularity conditions hold, the test statistic $J$ has an asymptotic $\chi^{2}$-distribution with $(3 p-1) n s+s^{2}$ degrees of freedom.

\subsubsection{Finite sample properties of the test}

Anderson and Vahid (1998) conducted several Monte Carlo experiments in order to evaluate the properties of the test when STAR nonlinearities are present. They restricted their attention to the bivariate and trivariate case with 0,1 or 2 and $0,1,2$ or 3 nonlinearities of the LSTAR type with the same known transition variable in the true data generating process (DGP), respectively. 
The simulations with sample sizes of 100 and 300 were conducted in each case. The bivariate LSTAR test performed well, especially in the sample size of 300, whereas the trivariate LSTAR test had difficulty distinguishing between the cases of 1, 2 and 3 common nonlinear components. It must be emphasized that the chosen nonlinear data generating processes were close to linear because of the small values of the $\gamma$ parameters. When performed on DGPs with higher $\gamma$ values, the tests performed substantially better.

\section{An application to the real Tolar - Euro exchange rate}

As an application of the described modelling approach, a three-variable vector autoregressive (VAR) models of the consumer price index for Slovenia, consumer price index of another country and the nominal exchange rate between the currencies of both countries are discussed. Therefore, this investigation applies the common nonlinearities techniques to small models of the real exchange rate, decomposed into its three components, domestic prices $\left(P_{t}\right)$, foreign prices $\left(P_{t}^{*}\right)$ and the nominal exchange rate $\left(S_{t}\right)$. The models have been applied to 5 most important foreign trade partners of Slovenia, namely Germany, Italy, France, Austria and Croatia.

Monthly data for the period from January 1988 till December 2003 were obtained from the Bank of Slovenia and from the Statistical Office of the Republic of Slovenia. Due to the fact that Slovenia declared independence in June 1991 and introduced its own currency (Tolar) in October of the same year, only the data for the period from January 1993 till December 2003, when Tolar was already an established currency, were used in the study. In the case of Croatia, the period under investigation was shortened additionally because of the war. Only the data from April 1995 till December 2003 were taken into account. The econometric model employs variables expressed in growth rates with the help of the logarithmic transformation, therefore small letters are used to denote the transformed variables.

In a preliminary specification, all equations were modelled as linear relationships. This simplifies the search for an appropriate nonlinear specification. Firstly, unit root tests were applied to the variables $p_{t}, p_{t}^{*}$ and $s_{t}$ for each of the countries. All of the variables turned out to be integrated of order 1, or I(1). Next, cointegration tests were performed and the linear vector error correction models (VECM) were specified. The null hypothesis of no cointegrating relations could not be rejected only in case of Croatia, therefore a linear VAR model in the differenced variables $\Delta p_{t}, \Delta p_{t}^{*}$ and $\Delta s_{t}$ was specified. Orthogonal seasonal dummy variables, denoted by $d 1$ to $d 12$, were introduced into some of the models to reduce the autocorrelation effects.

\subsection{Linearity test results}

In order to improve specification, we investigated the influence of nonlinearities, which we assumed to be of the smooth transition kind. For this purpose, we tested the null hypothesis of linearity against the alternative of a smooth transition autoregressive model for each of the equations and each of the possible transition variables in turn. The values of the F-statistic (and the corresponding p-values in brackets) are given in Table 1 below. The cointegrating equations are denoted by $c e 1$ and $c e 2$. 
Table 1: F-values (and p-values) for testing linearity against STR.

\begin{tabular}{|c|c|c|c|}
\hline \multicolumn{4}{|c|}{ Germany } \\
\hline transition variable & $\Delta p$ equation & $\Delta p^{*}$ equation & $\Delta s$ equation \\
\hline$c e 1_{t}$ & $1.642(0.039)$ & $\overline{1.072(0.393)}$ & $\overline{~ 4.178(0.000)}$ \\
\hline$\Delta p_{t-1}$ & $1.135(0.321)$ & $1.251(0.211)$ & $1.819(0.017)$ \\
\hline$\Delta p_{t-1}^{*}$ & $0.847(0.699)$ & $0.567(0.965)$ & $1.383(0.124)$ \\
\hline$\Delta s_{t-1}$ & $1.397(0.117)$ & $0.995(0.492)$ & $2.612(0.000)$ \\
\hline$\Delta p_{t-2}$ & $1.936(0.009)$ & $0.554(0.971)$ & $2.566(0.000)$ \\
\hline$\Delta p_{t-2}^{*}$ & $1.034(0.440)$ & $1.432(0.101)$ & $0.791(0.772)$ \\
\hline$\Delta s_{t-2}$ & $1.601(0.047)$ & $0.684(0.889)$ & $4.175(0.000)$ \\
\hline$\Delta p_{t-3}$ & $0.711(0.864)$ & $1.022(0.457)$ & $2.037(0.006)$ \\
\hline$\Delta p_{t-3}^{*}$ & $1.387(0.122)$ & $1.264(0.200)$ & $1.352(0.141)$ \\
\hline$\Delta s_{t-3}$ & $1.146(0.309)$ & $0.456(0.993)$ & $3.471(0.000)$ \\
\hline \multicolumn{4}{|c|}{ Italy } \\
\hline transition variable & $\Delta p$ equation & $\Delta p^{*}$ equation & $\Delta s$ equation \\
\hline$c e 1_{t}$ & $1.480(0.125)$ & 0.898 & $3.104(0.000)$ \\
\hline$\Delta p_{t-1}$ & $1.123(0.347)$ & $1.558(0.098)$ & $1.494(0.120)$ \\
\hline$\Delta p_{t-1}^{*}$ & $2.797(0.001)$ & $2.757(0.001)$ & $1.809(0.042)$ \\
\hline$\Delta s_{t-1}$ & $1.681(0.065)$ & $1.022(0.442)$ & $2.248(0.009)$ \\
\hline \multicolumn{4}{|c|}{ France } \\
\hline transition variable & $\Delta p$ equation & $\Delta p^{*}$ equation & $\Delta s$ equation \\
\hline$c e 1_{t}$ & 1.213 & 0.6 & $\overline{000)}$ \\
\hline$\Delta p_{t-1}$ & 0.878 & 0.9 & $.160)$ \\
\hline$\Delta p_{t-1}^{*}$ & $0.665(($ & $1.3710(0.139)$ & $0.919)$ \\
\hline$\Delta s_{t-1}$ & $1.210(0.253)$ & 0.7 & $.001)$ \\
\hline$\Delta p_{t-2}$ & $1.276(0.199)$ & .806) & $.300)$ \\
\hline$\Delta p_{t-2}^{*}$ & $1.022(0.460)$ & 0.8 & $.887)$ \\
\hline$\Delta s_{t-2}$ & $1.857(0.017)$ & $0.939((-2)$ & $0.000)$ \\
\hline$\Delta p_{t-3}$ & $1.112(0.351)$ & $0.776(($ & $0.003)$ \\
\hline$\Delta p_{t-3}^{*}$ & $0.861(0.677)$ & & $.220)$ \\
\hline & $1.150(0.310)$ & $0.843(0.701)$ & $2.045(0.007)$ \\
\hline \multicolumn{4}{|c|}{ Austria } \\
\hline transition variable & $\Delta p$ equation & $\Delta p^{*}$ equation & $\Delta s$ equation \\
\hline$c e 1_{t}$ & $1.392(0.153)$ & $1.002(0.468)$ & $0.001)$ \\
\hline$c e 2_{t}$ & $2.619(0.001)$ & $1.712(0.043)$ & $0.000)$ \\
\hline$\Delta p_{t-1}$ & $1.284(0.216)$ & $1.207(0.272)$ & $1.817(0.034)$ \\
\hline$\Delta p_{t-1}^{*}$ & $1.356(0.172)$ & $1.146(0.323)$ & $1.655(0.061)$ \\
\hline$\Delta s_{t-1}$ & $1.765(0.041)$ & $1.355(0.173)$ & $4.024(0.000)$ \\
\hline \multicolumn{4}{|c|}{ Croatia } \\
\hline transition variable & $\Delta p$ equation & $\Delta p^{*}$ equation & $\Delta s$ equation \\
\hline$\Delta p_{t-1}$ & $1.222(0.282)$ & $1.432(0.166)$ & $0.840(0.617)$ \\
\hline$\Delta p_{t-1}^{*}$ & $0.755(0.704)$ & $1.110(0.365)$ & $2.719(0.004)$ \\
\hline$\Delta s_{t-1}$ & $0.616(0.834)$ & $0.638(0.814)$ & $4.208(0.000)$ \\
\hline
\end{tabular}


Table 2: Common nonlinearities test for Italy and Austria.

\begin{tabular}{|c||cc|}
\hline \multicolumn{3}{|c|}{ Italy } \\
\hline \multicolumn{3}{|c|}{ tvar: $\Delta p_{t-1}^{*}$} \\
\hline$s$ & p-value & df \\
\hline \hline 1 & 0.230 & 10 \\
2 & 0.033 & 22 \\
3 & 0.000 & 36 \\
\hline
\end{tabular}

\begin{tabular}{|c||cc|}
\hline \multicolumn{3}{|c|}{ Austria } \\
\hline \multicolumn{3}{|c|}{ tvar: $c e 2_{t}$} \\
\hline$s$ & p-value & df \\
\hline \hline 1 & 0.262 & 13 \\
2 & 0.079 & 28 \\
3 & 0.001 & 45 \\
\hline
\end{tabular}

The goal of the study was to obtain a model with only one common nonlinear component, because in this way a parsimonious specification is achieved. Obviously, the necessary condition for the existence of such a model is a transition variable for which the null hypothesis of linearity is rejected for every equation in the model. There are only two variables that comply with this condition in our case, namely the variable $\Delta p_{t-1}^{*}$ in the model for Italy and the variable $c e 2_{t}$ in the model for Austria. The significance level of $5 \%$ is assumed. Table 2 shows the results of the common nonlinearities test for both of the mentioned transition variables.

In accordance with the theory from the previous section, $s$ is equal to 1 for Italy and 2 for Austria. The number of common nonlinear components, which is determined by the formula $n-s$, takes the value of 2 for Italy and the value of 1 for Austria. Consequently, we shall concentrate on the model for Austria from now on.

\subsection{Estimated model for Austria}

The full information maximum likelihood (FIML) estimator was employed to allow for correlated residuals in different equations. Due to occasional problems with convergence of the nonlinear optimization procedure some experimentation to find appropriate starting values was required. The final set of estimates obtained for the parameters of the nonlinear multivariate logistic smooth transition autoregressive model of the Slovenian Tolar versus the Austrian Schilling, respectively Euro, can be found below. The estimated standard errors are given in brackets.

Common nonlinear component:

$$
\begin{aligned}
c o m_{t}= & \frac{1}{1+e^{-53.0123\left(c e 2_{t}+0.0993\right)}} * \\
* & (29.608) \quad(0.016) \\
& \left(0.00315-0.0717 \cdot c e 1_{t}-0.2177 \cdot c e 2_{t}+\right. \\
& \quad(0.096)(0.0196) \quad(0.0613) \\
+ & \left.0.4792 \cdot \Delta p_{t-1}^{*}+0.1063 \cdot \Delta s_{t-1}\right) \\
& (0.2486)
\end{aligned}
$$


First equation:

$$
\begin{aligned}
& \Delta p_{t}=0.0384+0.0388 \cdot c e 1_{t}+0.1506 \cdot c e 2_{t}-0.6346 \cdot \Delta p_{t-1}^{*}+ \\
& (0.0091)(0.0188) \quad(0.0663) \quad(0.2491) \\
& +\quad 0.0086 \cdot d 1_{t}+0.0055 \cdot d 2_{t}+0.0053 * d 3_{t}+0.0053 \cdot d 4_{t}+ \\
& \begin{array}{lll}
(0.0015) \quad(0.0020) \quad(0.0018) \quad(0.0015)
\end{array} \\
& +\quad 0.0056 \cdot d 5_{t}+0.0033 \cdot d 7_{t}+0.0057 \cdot d 9_{t}+0.0036 \cdot d 10_{t}+ \\
& \begin{array}{lll}
(0.0015) \quad(0.0019) \quad(0.0016) \quad(0.0025)
\end{array} \\
& +\quad 0.0047 \cdot d 11_{t}+0.0025 \cdot d 12_{t}+\operatorname{com}_{t} \\
& (0.0015) \quad(0.0018)
\end{aligned}
$$

Second equation:

$$
\begin{aligned}
\Delta p_{t}^{*}= & 0.0031+0.0072 \cdot c e 2_{t}+0.0526 \cdot \Delta s_{t-1}+0.0016 \cdot d 2_{t}+ \\
& (0.0010)(0.0052) \quad(0.0390) \quad(0.0013) \\
+ & 0.0013 \cdot d 7_{t}-0.0027 \cdot d 9_{t}+0.0620 \cdot \mathrm{com}_{t} \\
& (0.0009) \quad(0.0009) \quad(0.0147)
\end{aligned}
$$

Third equation:

$$
\begin{aligned}
\Delta s_{t}= & 0.0620+0.1642 \cdot c e 1_{t}+0.4629 \cdot c e 2_{t}-1.1531 \cdot \Delta p_{t-1}^{*}+ \\
& (0.0147)(0.0305) \quad(0.1055) \quad(0.4513) \\
+ & 0.5001 \cdot \Delta s_{t-1}-0.0024 \cdot d 5_{t}-0.0022 \cdot d 7_{t}+1.9444 \cdot c o m_{t} \\
& (0.0905) \quad(0.0017) \quad(0.0020)
\end{aligned}
$$

Note that both of the crucial parameters in the common nonlinear part, $\gamma$ and $c$, are significant at the $10 \%$ level. The $\gamma$ value of approximately 53 indicates rapid transition between the two extreme regimes.

A comparison of single equations from the linear and nonlinear system (Table 3 ) reveals an increase in explanatory power for equations 1 and $3\left(R^{2}\right.$ increases from 0.62 to 0.65 and from 0.67 to 0.76 , respectively) and a decrease in the standard error of regression from 0.0039 to 0.0038 and from 0.0051 to 0.0043 , respectively. For equation 2 , the situation is just the opposite. There is a decrease in explanatory power, while the standard error of regression stays the same. It should be emphasized that there are better ways to analyze systems of equations than single-equation comparison. If we compare the value of log-likelihood for both systems, we can observe that it is higher for the nonlinear system (1668.52 as compared to 1649.64 for the linear system), indicating an improvement in specification.

\section{Conclusion}

The obtained real exchange rate model of the Slovenian Tolar versus the Austrian Schilling, respectively Euro, contains only one common nonlinear component, as desired. On the 
Table 3: Comparing equations in the linear and nonlinear system.

\begin{tabular}{|c||ccc|}
\hline \multicolumn{4}{c|}{ Linear system } \\
\hline & $\Delta p$ equation & $\Delta p^{*}$ equation & $\Delta s$ equation \\
\hline \hline$R^{2}$ & 0.62 & 0.22 & 0.67 \\
S.E. & 0.0039 & 0.0028 & 0.0051 \\
\hline
\end{tabular}

\begin{tabular}{|c|ccc|}
\hline \multicolumn{4}{c|}{ Nonlinear system } \\
\hline & $\Delta p$ equation & $\Delta p^{*}$ equation & $\Delta s$ equation \\
\hline \hline$R^{2}$ & 0.65 & 0.17 & 0.76 \\
S.E. & 0.0038 & 0.0028 & 0.0043 \\
\hline
\end{tabular}

other hand, the models for Germany, Italy, France and Croatia cannot be adequately described with the help of only one type of nonlinearity. One of the possible explanations for such results could be the late accession of Austria to the European Union. Austria joined EU in the year 1995, whereas Germany, Italy and France were already member states in the year 1993, when we started our investigation. The process of Austria's EU accession had a deep impact on its economic structure and the relation to its neighbour states. In particular prices have been severely affected. These adjustments together with those ongoing in neighbouring Slovenia seem to be captured by a common nonlinear factor in the components of the real exchange rate. It concerns especially the effects of the lagged Austrian inflation rate and the nominal exchange rate, besides the cointegration terms. This lends economic support to the specification of a multivariate logistic STAR model with only one common nonlinear component.

\section{References}

[1] Anderson, H.M. and Vahid, F. (1998): Testing multiple equation systems for common nonlinear components. Journal of Econometrics, 84, 1-36.

[2] Camacho, M. (2004): Vector smooth transition regression models for US GDP and the composite index of leading indicators. Journal of Forecasting, 23, 173-196.

[3] Granger, C.W.J. and Teräsvirta, T. (1993): Modelling Nonlinear Economic Relationships. Oxford: Oxford University Press.

[4] Greene, W.H. (2003): Econometric Analysis. New Jersey: Prentice Hall.

[5] Kavkler, A. (2006): Econometric models of smooth transition, doctoral disertation. Vienna: Vienna university of technology.

[6] Krolzig, H.M. (1997): Markov-Switching vector autoregressions-modelling, statistical inference and applications to business cycle analysis. Lecture Notes in Economics and Mathematics, 454. Berlin: Springer Verlag. 
[7] Luukkonen, R., Saikkonen, P. and Teräsvirta, T. (1998a): Testing linearity against smooth transition autoregressive models. Biometrika, 75, 491-499.

[8] Luukkonen, R., Saikkonen, P. and Teräsvirta, T. (1998b): Testing linearity in univariate time series. Scandinavian Journal of Statistics, 15, 161-175.

[9] Teräsvirta, T. (1998): Modelling economic relationships with smooth transition regression. In A. Ullah and D.E. Giles (Eds): Handbook of Applied Economic Statistics, 507-552. New York: Marcel Dekker.

[10] Teräsvirta, T. (1994): Specification, estimation and evaluation of smooth transition autoregressive models. Journal of the American Statistical Association, 89, 208218.

[11] Tsay, R.S. (1998): Testing and modeling multivariate threshold models. Journal of the American Statistical Association, 93, 1188-1202.

[12] van Dijk, D. (1999): Smooth Transition Models: Extensions and Outlier Robust Inference. Amsterdam: Tinberg Institute.

[13] Weise, C.L. (1999): The asymmetric effects of monetary policy: A nonlinear vector autoregression approach. Money, Credit and Banking, 31, 85-108.

[14] White, H. (2001): Asymptotic Theory for Econometricians. San Diego: Academic Press.

[15] Wooldridge, J.M. (1994): Estimation and inference for dependent processes. In R.F. Engle and D.L. McFadden (Eds): Handbook of econometrics, Vol. 4, 2649-2738. Amsterdam: North - Holland. 